Anuario Latinoamericano Ciencias Políticas

y Relaciones Internacionales

vol. 1, 2014

p. $45-69$

\title{
¿Sur-Sur contra Norte-Sur? La política brasileña extraregional bajo la presidencia de Lula da Silva (2002-2011)
}

\author{
Klaus Bodemer \\ GIGA INSTITUTO DE ESTUDIOS LATINOAMERICANOS, \\ HAMBURGO, ALEMANIA \\ klaus.bodemer@giga-hamburg.de
}

\section{RESUMEN}

Iniciada ya durante el segundo gobierno de F. H. Cardoso, la política exterior brasileña, bajo su sucesor L. I. Lula da Silva, experimentó un cambio de rumbo con el lema de "autonomía por diversificación". Las características centrales de esta reorientación fueron un reclamo más pronunciado del país por un liderazgo regional y la profundización de las relaciones Sur-Sur con el objetivo de fortalecer la capacidad negociadora del Sur y alcanzar un orden mundial más justo $y$ equilibrado. Los resultados de esta política son, tanto al nivel regional como internacional, más bien mezclados. Al nivel regional, se muestra que el poder posicional no se traduce automáticamente en un poder de influencia; al nivel internacional, el país fue capaz de formar alianzas, pero su "power of outcome" fue más bien modesto y el intento de presentarse como "abogado del Sur", por lo menos, discutible.

PALABRAS CLAVE: Brasil, relaciones Sur-Sur, politica exterior, Lula da Silva.

\section{SUMMARY}

During the second term of F. H. Cardoso and under the government of his successor L. I. da Silva, the foreign policy of Brazil underwent a change of course, both in domestic policy as well as in the international arena. The main characteristics of this change were a more pronounced claim for a "cooperative leadership" in the region and the deepening of the South-South relations with the objective to strengthen the negotiation capacity of the South and to attain a more just and balanced international regime. The results are mixed. On the regional level, it is clear that the positional power does not translate automatically into the power of influence; on the international level, the Brazilian diplomacy was able to build alliances, but its power of outcome was rather modest and the intention to present itself as "Advocate of the South", at least, questionable.

KEYWORDS: Brazil, South-South relations, foreign policy, Lula da Silva. 
América Latina: cambios a nivel regional y en su inserción internacional

\section{De la "autonomía por distancia" a "la autonomía por participación"}

La política exterior brasileña ha seguido durante años una estrategia de inserción muy selectiva y cautelosa en el mercado mundial, reflejo del concepto cepalino de substitución de importaciones (ISI). En reacción a la "década perdida" (los años 80) y la segunda ola de integración, el llamado "regionalismo abierto", ya bajo de la presidencia de Fernando Collor de Mello (1990-1992) y su sucesor Itamar Franco (1992-1994), Brasil se ha despedido de la estrategia ISI y de la vieja cultura del "inward-looking policymaking" (Baumann 2010: 33). El concepto de "autonomía por distancia" fue reemplazado en la política exterior por el de "autonomía por participación" (Vigevani y Cepaluni 2007: 283 y ss.; Facundes Vicentini y Reis da Silva 2010: 54). Collor de Mello bajó radicalmente las barreras aduaneras e inició un proceso de privatizaciones, acompañado por una política exterior que se orientó - otra vez más en la historia brasileña - a las demandas de EE.UU. y la U.E. Los ataques terroristas del 11 de septiembre de 2001 y sus repercusiones internacionales subrayaron, además, la necesidad de redefinir la seguridad internacional, fortalecer el multilateralismo y el orden internacional multipolar (Lessa 2010: 116). La política de estabilidad, cuyos rasgos se fundamentaron en el Plano Real, permitió, finalmente, al sucesor de Collor de Mello, el socialdemócrata Fernando Henrique Cardoso (1995-2002), avanzar hacia una nueva proyección internacional del país, hacer más atractiva la industria local y atraer inversiones exteriores directas. Como respuesta al acelerado proceso de la globalización, Cardoso confiaba en la configuración ideal de un nuevo orden multipolar y en el desarrollo progresivo de relaciones internacionales más justas y armónicas (Bernal Mesa 2010: 198). Con respecto a la región, Cardoso aplicó una cautelosa modificación de la política exterior brasileña propagando el liderazgo de su país en la región bajo el lema del binomio seguridad - estabilidad democrática, estableció estrechos vínculos con sus vecinos y actuó como mediador en conflictos (Gomes Saraiva 2007: 48). Cardoso negoció, además, sobre el ALCA con EE.UU. y con Europa sobre un acuerdo de asociación entre la U.E. y el Mercosur. Eso significaba comprometerse activamente con los Estados centrales del sistema internacional y cooperar con los regímenes internacionales en vigencia bajo el concepto de la "soberanía compartida" (Alden y Vieira 2007: 155; Vigevani y Cepaluni 2007: 300, 304). Cardoso buscó así un mayor reconocimiento del país, participando en la visión multilateralista del ex presidente Bill Clinton, basada en el neo-idealismo kantiano y un institucionalismo pragmático (Bernal Mesa 2008: 31).

Fue recién en su segundo mandato que Cardoso se ocupó más intensamente de una reorientación de la política exterior, reaccionando al hecho de que la consolidación del proceso de integración, sobre todo del Mercosur y su ampliación con UNASUR, fortaleció, bajo el lema de la "autono- 
mía por integración" (Oliveira 2005: 55), la posición negociadora del país en los diferentes foros multilaterales y su liderazgo en la región. Se trataba, sin embargo, no de un cambio radical de la política exterior brasileña, sino más bien de "una mudanza dentro de la continuidad" (Vigevani y Cepaluni 2007: 322).

\section{El cambio de rumbo en la política exterior brasileña bajo el gobierno de Lula da Silva}

El sucesor de Cardoso, Lula da Silva (2002-2011), del Partido Trabajador (PT), profundizó esta reorientación y expandió y reestructuró las relaciones exteriores, apuntando a nuevas agrupaciones bilaterales y regionales. Un primer paso fue una viva diplomacia de viajes. Sólo en el primer año de su mandato, Lula visitó 38 países. El objetivo principal de esta diplomacia de viajes fue una nueva geografía del comercio mundial que, según la convicción del nuevo gobierno brasileño, debería ajustarse mejor a los intereses y necesidades de los países del Sur. La cooperación con países emergentes importantes, como China, India y África del Sur, tenía en este contexto un peso particular. La idea subyacente era que a través de alianzas y de una cooperación eficaz se alcanzaría contar con suficientes recursos tecnológicos, fuerza de trabajo calificada y el capital necesario para disminuir la dependencia del país de las potencias del Norte.

Estas ambiciones en la política exterior brasileña fueron en última instancia un reflejo de un cambio estructural en la arena mundial, caracterizado por el empoderamiento de las regiones del Sur, lo que ha aportado a un power shift en la política internacional. Desde los años 90 , las regiones son cada vez más importantes como unidades de acción en el mundo (Katzenstein 2005). Países emergentes, como China, India, África del Sur o Brasil, juegan cada vez más un rol prominente en sus regiones, traspasando, además, esas fronteras y actuando como jugadores globales a nivel internacional. Estos poderes regionales parecen jugar de manera diferente en su entorno, utilizando estrategias variadas e implementando instrumentos que van desde medidas de confianza mutua y de políticas soft power hasta políticas coercitivas y de represión militar (como, por ejemplo, Rusia en Chechenia).

En lo que sigue quiero concentrarme en la política exterior extraregional Sur-Sur de Brasil durante las dos presidencias de Lula da Silva, dejando más bien al margen (pero no totalmente afuera, porque constituye la base de la proyección internacional) el rol que Brasil juega en su entorno subregional y regional. En cuanto a mi aproximación teórica, me apoyo, en primer lugar, en el enfoque neo-estructuralista y social-constructivista que toma como objeto de análisis la parte discursiva de la política.
¿Sur-Sur contra Norte-

Sur? La política brasileña

extraregional bajo la

presidencia de Lula

da Silva (2002-2011)

Klaus Bodemer 
América Latina: cambios a nivel regional y en su inserción internacional

\section{"Autonomía por diversificación" - Objetivos, líneas directrices y prioridades estratégicas de la política exterior de Lula da Silva}

Durante el gobierno de Fernando Henrique Cardoso predominaban en Itamaraty los llamados autonomistas, cuya prioridad era América del Sur. El discurso del Presidente apuntaba al destino de la nación; los socios privilegiados del país eran los países del Norte. La política exterior brasileña siguió entonces la tradición legalista que muchos años había determinado el comportamiento exterior del país. El núcleo de esta tradición era el respeto por las normas internacionales, por ejemplo del Tratado de Tlatelolco, y su lema era no desafiar la agenda internacional. Además, Cardoso le asignaba un rol fundamental al desarrollo y la capacidad tecnológica para poder competir internacionalmente.

Lula da Silva ha dado a la política exterior brasileña otros acentos. En vez de un enfoque más bien nacionalista, como el de Cardoso, su sucesor fue, en primer lugar, internacionalista. Los formuladores principales de esta política exterior fueron el Presidente mismo, seguido por el Canciller, el Embajador Celso Luiz Nunes Amorim, el Secretario General de Relaciones Exteriores, el Embajador Samuel Pinheiro Guimarães y el Asesor Especial para Asuntos Internacionales de la Presidencia, Marco Aurélio Garcia, en cierto sentido la "eminencia gris" del Presidente y su vínculo con el PT. Su rol como ideólogo y en parte también como responsable de la ejecución de ciertas líneas de la política exterior brasileña fue excepcional, cuestionando no pocas veces el "monopolio de facto" que la diplomacia de carrera bajo la tutela de Itamaraty ha ocupado durante décadas (Almeida 2006: 98).

Según Lula da Silva, los impactos más fuertes de la globalización se notan en el comercio exterior. Distanciándose en otros aspectos de la izquierda latinoamericana, el gobierno de Lula compartió la crítica de esta izquierda a los programas de ajuste bajo el régimen del "Consenso de Washington" y se refirió al "Consenso de Buenos Aires", un entendimiento entre Brasil y Argentina con el acento en asuntos económicos y sociales de la agenda internacional. Lula no compartió, sin embargo, la crítica a ultranza a la globalización de sus homólogos en Venezuela, Ecuador y Bolivia, sino que prefirió insertarse en la globalización de manera pragmática, haciéndola más justa, equilibrada y democrática, y aprovechando las ventajas que se ofrecen con tal estrategia (Almeida 2006: 100 y s; Cervo 2010: 8).

Un concepto núcleo de la nueva política exterior brasileña fue el de "Estado logístico", comprendido como una guía estratégica para Brasil en la arena internacional. Según Cervo el Estado "desempeña el papel de promotor y abanderado de las iniciativas de otros actores económicos y sociales" (Cervo 2010: 10). El concepto incluye, además, dos dimensiones: primero, ciertas percepciones sobre la globalización, la interdependencia global y el regionalismo como características del mundo actual; segundo, la convicción de la disminución de la estabilidad hegemónica de los EE.UU. y la emergencia de un mundo basado en el multilateralismo y una nueva estratificación de poder, 
donde Brasil, como una potencia emergente, tiene que jugar un rol importante. El concepto del "Estado logístico" permite a Brasil, como subraya Bernal Mesa (Bernal Mesa 2010: 201 y s.), desacoplarse de un mundo anclado en las categorías centro-periferia, colocarse en una posición media, ocupar un rol de liderazgo en América del Sur y asumir, junto con otros system affecting countries como India, China y África del Sur, el rol de un liderazgo colectivo con el objetivo de alcanzar un orden internacional más justo y democrático (Bernal Mesa 2010: 203).

Los principios fundamentales que orientaron la política exterior de Lula da Silva fueron la preservación de la soberanía nacional, la no injerencia en asuntos internos, el fortalecimiento del Estado en la política exterior, un multilateralismo recíproco, compatible con el desarrollo (Oliveira 2005: 56), y la ampliación de la "autonomía por participación", que había predominado durante los gobiernos de Franco y Cardoso, a una "autonomía por diversificación” (Duarte Villa y Trindade Viana 2010: 92 y s; Vigevani y Cepaluni 2007: 281-284).

Conceptualmente, Lula da Silva y sus formuladores de la política exterior compartieron la visión de Joseph Nye de un orden multilateral que permita coordinar el pensamiento liberal de Celso Lafer, canciller de Cardoso, con el realismo del nuevo gobierno (Bernal Mesa 2010: 199). En general, la nueva dinámica se reflejó en una política exterior más marcada y auto-consciente. Internacionalmente, la diplomacia brasileña se concentró en la política financiera y el comercio internacional y se apoyó, en primer lugar, en las relaciones económicas y políticas ampliadas con los otros países emergentes. Al nivel regional, la diplomacia privilegió las cuestiones de seguridad. Ambas estrategias sirvieron, en cierto sentido, como medidas para proponerse como miembro permanente en el Consejo de Seguridad de las Naciones Unidas. Aprovechando la difusión de poder en la política internacional, Brasil apuntaba con todo eso a una bifurcación: intentó ser, al mismo tiempo, una parte del club de los ricos, es decir de los países OCDE, socio de los países emergentes, agrupados en el IBSA y los países BRICS, vocero de América del Sur y representante de los intereses del Tercer Mundo, incluidos los non-system affecting countries frente a los países OCDE y, finalmente, un país de peso que fomenta decididamente la cooperación Sur-Sur ${ }^{1}$. Para implementar una política exterior tan compleja y ambiciosa, Lula da Silva y su equipo han practicado una diplomacia asertiva y de geometría variable con diferentes vertientes.

1 El país es miembro de una multitud de organizaciones y regímenes internacionales y su diplomacia persigue un comportamiento dinámico, articulando sus intereses como global jugador en asuntos como las finanzas globales, el comercio internacional, el medio ambiente y el cambio climático, la seguridad internacional y la reforma del sistema de Bretton Woods, demostrando de esta manera una creciente autoconciencia y un visible posicionamiento en la arena internacional. Su lucha por un sitio permanente en el Consejo de Seguridad está acompañada por el pedido de una reforma substancial de la Carta de las Naciones Unidas. Con el resurgimiento del debate nuclear, Lula desafió la agenda internacional.
¿Sur-Sur contra NorteSur? La política brasileña extraregional bajo la presidencia de Lula da Silva (2002-2011)

Klaus Bodemer 
América Latina: cambios a nivel regional y en su inserción internacional

\section{La política subregional y regional como hegemonía cooperativa}

Con respecto a la región, Lula siguió apostando a la integración regional, pero con una institucionalidad mínima y priorizando la extensión del Mercosur en vez de su profundización, lo que ha culminado, por fin, en la creación de UNASUR. En ese sentido, su diplomacia se veía enfrentada a dos desafíos: primero, impedir que los tratados de libre comercio bilaterales se vuelvan el patrón dominante en la región y, segundo, frenar la diplomacia petrolera de Chávez.

El tema más discutido en este contexto es si Brasil ha alcanzado bajo el gobierno de Lula definitivamente un rol de liderazgo en la región. Como se verá en detalle más abajo, no hay dudas de que los datos duros están apoyando esta hipótesis. La aceptación de su rol de líder por los vecinos (todos los países latinoamericanos con la excepción de dos: Ecuador y $\mathrm{Su}$ rinam) es, sin embargo, un problema. Algunos observadores hablan, por lo tanto, de un liderazgo sin seguidores (Malamud 2010). Si nos preguntamos si Lula mismo se ha expresado sobre el tema del "liderazgo" y/o "hegemonía”, encontramos respuestas ambivalentes. Tanto él mismo, como su asesor principal en asuntos exteriores, Marco Aurélio Garcia, han declarado repetidas veces que Brasil no está aspirando a tal rol de liderazgo en América del Sur. Por otro lado, mirando los hechos, me parece indudable que la convicción básica, el leitmotiv de la política exterior brasileña bajo los dos turnos de la presidencia de Lula da Silva fue que sin liderazgo regional (más allá del Mercosur) no hay un protagonismo internacional. Además, Lula ha sostenido que dentro del Mercosur la cooperación con Argentina, su competidor mayor, es fundamental y debe por eso ser fuerte. La integración regional tenía para Lula, por lo tanto, en primer lugar, un valor instrumental y estratégico, mientras el objetivo a largo plazo era la inserción del país como uno de los países líderes en el ámbito internacional. Este proyecto incluyó nuevos actores y metas. Tres fueron sus prioridades: primero, la estabilidad política regional que exige, entre otros, una política de defensa articulada; segundo, una política extra-regional y, tercero, el fomento de exportaciones de bienes industriales, una infraestructura sólida y una matriz energética sustentable. El gobierno ha presentado con respecto a estas prioridades un plan llamado "Brasil 3 Tiempos" (Santos 2011: 207 y s.). En analogía a la política interna de "fome zero" se ha hablado del "fome zero internacional" como objetivo a largo plazo, que comienza con la reforma de las estructuras financieras internacionales existentes, es decir, del FMI, el Banco Mundial y el sistema de las Naciones Unidas, hacia regímenes más equilibrados y más participativos.

Según el rumbo extraregional favorecido, los flujos comerciales exteriores de Brasil se reorientaron decididamente desde América hacia al resto del mundo. La Unión Europea ocupaba el primer puesto como socio comercial, pero fue sobrepasada por Asia Oriental. Su tercer socio era EE.UU. y, des- 
pués, en orden descendente, Mercosur, África y Medio Oriente. Los países del Mercosur no presentan más que una octava parte del total. Brasil mantiene un superávit comercial con la U.E., EE.UU., el Mercosur y el Oriente Medio, mientras su balanza es deficitaria con Asia y África.

Haciendo un balance de la política regional de Lula, se la puede caracterizar como hegemonía cooperativa. Este comportamiento tiene - siguiendo a Pedersen - "la ventaja de difusión". Según eso, la constitución de y la participación en instituciones y los mecanismos regionales le sirven a Brasil para la expansión de valores, ideas y proyectos (Pedersen 2002: 681). De esta manera, Brasil apunta al rol de un hegemón benevolente y utiliza para este fin los proyectos de integración regional como plataforma para un comportamiento cooperativo (Burges 2008: 75). Las causas de esta actitud son, ante todo, tres: primero, el país no dispone de los recursos económicos, políticos y militares suficientes para desempeñar una hegemonía coercitiva; segundo, el comportamiento cooperativo y la inclusión de otros Estados permite el acceso a otros mercados, y, finalmente, una región más integrada permite a los países participantes negociar con los países del Norte sobre una base más equilibrada. En suma, el comportamiento cooperativo de Brasil en la región le ha ayudado a ejercer un rol de liderazgo en los hechos. Las relaciones con Argentina y Venezuela son un ejemplo al respecto. La inclusión de este último país en el Mercosur y UNASUR puede ser entendida, por lo tanto, como intento de domesticar sus ambiciones como potencia regional (Seifert 2009: 60). Brasil dispone de la necesaria power aggregation capacity, es decir de la capacidad de motivar a otros países a participar en los esquemas de integración más ambiciosos (Seifert 2009: 67). Esta política le sirve a Brasil, además, para actuar como un factor de estabilización, un elemento visto positivamente, sobre todo, desde el Norte, es decir, por EE.UU. y la U.E.

\section{Hacia un nuevo protagonismo: la política brasileña Sur-Sur extra-regional}

Entre los socios extraregionales podemos diferenciar, siguiendo a Miriam Gomes Saraiva, entre system-affecting states y non system-affecting states (Gomes Saraiva 2007). Con respecto a los primeros, se trata de países que disponen de menos recursos y capacidades que los países grandes, pero que pueden influir en la agenda internacional, por ejemplo, en una actuación concertada. Por lo común, estos países se ocupan de un perfil internacional integrativo y reconocen instituciones multilaterales como arenas adecuadas para la interacción internacional. Non system-affecting states son aquellos países que no cumplen estos requisitos. Entre ellos se encuentra la mayoría de los socios de Brasil en África, pero también en Asia y el Medio Oriente.

Uno de los países más importantes del primer grupo es China. Sus relaciones con Brasil se concentran en el área económica, mientras que la co-
¿Sur-Sur contra Norte-

Sur? La política brasileña

extraregional bajo la

presidencia de Lula

da Silva (2002-2011)

Klaus Bodemer 
América Latina: cambios a nivel regional y en su inserción internacional operación política muchas veces no funciona por los intereses divergentes. La cooperación política es, por otro lado, más importante en el caso de India y África del Sur, mientras que la cooperación económica es en estos casos más reducida debido a la falta de complementariedad de las economías.

En lo que sigue me concentraré en la cooperación brasileña en el marco de instituciones plurinacionales y multilaterales, en concreto: el IBSA, los BRICS, la OMC y el G20.

\section{Brasil en el Foro IBSA}

El foro plurinacional del Sur más importante es IBSA, fundado por iniciativa brasileña en junio de 2003 con la Declaración de Brasilia. Los tres países miembros - India, Brasil y África del Sur - disponen de características comunes con respecto a su importancia regional, su creciente capacidad económica, su pretensión de participación internacional y su adhesión a la comunidad occidental de valores (Gratius y de Sousa 2007: 2 y s.). Los tres países comparten la dinámica de una población multiétnica y multicultural que ha alentado en cada uno de ellos políticas de inclusión social y la priorización de tales temáticas en la agenda de IBSA bajo el lema de "crecimiento económico y equidad social” (White 2006: 1). Según el documento fundacional, Declaración de Brasilia, los principios fundamentales del Foro fueron: el respeto por las reglas del derecho internacional, el fortalecimiento del sistema de las Naciones Unidas y del Consejo de Seguridad y la priorización de la diplomacia como medio para mantener la paz y la seguridad internacional. El acuerdo tripartito IBSA se comprende como una plataforma de diálogo entre los tres continentes del Tercer Mundo (África, Asia y América Latina), que tiende al fortalecimiento de la capacidad negociadora de los tres, una mayor participación y derechos de decisión en las instituciones de las Naciones Unidas y la OMC. El espectro de los temas de la cooperación incluye cuestiones de comercio y política económica, como el comercio de cargas y medicamentos genéricos (el derecho de patentes, la propiedad intelectual), energía (incluida bioenergía), la ampliación y el facilitamiento del comercio internacional, el fomento de microempresas en la industria agraria, la educación y la formación científica. Con respecto a la reforma del Consejo de Seguridad, los tres países abogaron por la ampliación de sus miembros, tanto en cuanto a los permanentes como a los no permanentes, incluyendo a los países en desarrollo. Brasil, India y África del Sur estuvieron de acuerdo también en fortalecer la eficiencia de la Asamblea General y del Consejo Económico y Social. Los cancilleres de los tres países subrayaron, además, su intención de dar un mayor ímpetu a la cooperación en el área de tecnología, computer science y agricultura (Declaración de Brasilia, 2010). Fueron incluidos, además, temas como la energía renovable y las patentes farmacéuticas.

¿Por qué cooperan estos tres países? La respuesta no es unánime. Hay voces que dicen que para países con capacidades materiales semejantes 
sería mejor competir que cooperar (Viola/Pio 2003). El multilateralismo Sur-Sur fue comprendido por los tres socios como una salida viable para enfrentar las adversidades sistémicas en un mundo post 9/11, caracterizado por un acentuado unilateralismo por parte de la superpotencia, los EE.UU, bajo la administración Bush Jr. Además, la aspiración de transformar el país en un actor relevante en la política internacional está fuertemente enraizada tanto en la historia diplomática de India como en la de Brasil. Un caso diferente es África del Sur, cuya inserción internacional comenzó recién a partir de 1990, es decir, luego del final del apartheid. Con respecto al contenido, los tres socios privilegiaron una concertación de posiciones en el ámbito internacional en tres áreas: seguridad, derechos humanos y desarrollo; y alcanzaron, según Freitas Rodrigues, una convergencia de posicionamientos del $88,50 \%$ en las votaciones en la Asamblea General de las Naciones Unidas (Freitas Rodrigues 2010: 59). Brasil se encuentra en coaliciones tanto con India como con África del Sur, pero África del Sur no se asocia con India sin la participación brasileña. Otra característica es que las relaciones entre Brasil e India, así como entre India y África del Sur, son de una baja complementariedad bilateral, reflejada en bajas relaciones comerciales y estrategias políticas conjuntas frente a una alta complementariedad multilateral en la ONU y la OMC. Los tres países son, además, competidores en varios productos y servicios en el mercado mundial y su comercio bilateral está dificultado por las grandes distancias, con el resultado de que el comercio mutuo equivale apenas al $2 \%$ del intercambio total. Sin embargo, tanto el comercio trilateral como el de los tres países con sus bloques respectivos ha aumentado significativamente desde la creación del Grupo IBSA en 2003 (Freitas Rodrigues 2010: 62 y s.). En general, la división entre una baja complementariedad bilateral y una alta complementariedad multilateral puede ser comprendida como un incentivo para la formulación de una agenda común del Foro del Diálogo IBSA. La experiencia muestra, además, que la influencia de posiciones concertadas en instituciones multilaterales es más eficiente si se trata de instituciones con dispersión de poder, como por ejemplo la OMC, y menos eficiente en un organismo con un poder concentrado, como el Consejo de Seguridad. En este último caso, el objetivo de la reforma corre el riesgo de quedar en pura retórica (Freitas Rodrigues 2010: 64). La verdadera fortaleza del Foro IBSA es su membrecía estrecha y el alto grado de posiciones comunes en los foros internacionales. Esto y el peso combinado de los tres miembros del Foro no tienen precedente en ejemplos anteriores de cooperación Sur-Sur. Los intentos anteriores se habían caracterizado por una membrecía amplia y amorfa, una entonación muy ideológica y una programática muy difusa. IBSA se ve, sin embargo, no como una alternativa a iniciativas anteriores Sur-Sur, sino más bien como un paso constructivo hacia una cooperación más amplia y profunda, enfrentando temas relevantes de la agenda internacional desde la perspectiva de desarrollo (White 2006: 5).
¿Sur-Sur contra NorteSur? La política brasileña extraregional bajo la presidencia de Lula da Silva (2002-2011)

Klaus Bodemer 
América Latina: cambios a nivel regional y en su inserción internacional
Para diferenciarse de iniciativas anteriores de cooperación Sur-Sur, el Foro necesitaba un foco claro y un plan de acción detallado. Este último fue elaborado y promulgado en la primera reunión de la Comisión Trilateral en Nueva Delhi en 2004. Seis áreas de cooperación fueron establecidas allí: (1) multilateralismo y cooperación multilateral, (2) paz y seguridad, (3) terrorismo, (4) globalización, (5) desarrollo sostenible y (6) desarrollo social. Estas áreas amplias fueron concretizadas en un Plan de Acción que definió sectores específicos con la intención de profundizar las relaciones trilaterales al respecto. El Plan de Acción fue tratado en 13 Grupos de Trabajo según departamentos gubernamentales existentes o prioridades domésticas, incluyendo los siguientes sectores: transporte (aviación civil y transporte marítimo), turismo, comercio e inversiones, infraestructura, job creation y PYMES, ciencia y tecnología, sociedad de información, salud, energía, defensa, educación, reducción de la pobreza y del hambre y, finalmente, gobernanza. Como se puede ver, este Plan de Acción con sus amplios subtemas careció de un foco estratégico preciso y corrió el riesgo de no llegar a resultados concretos.

Brasil fue el promotor de IBSA y se ha ido transformando también en el líder del Foro. Actualmente, los problemas centrales que tiene que enfrentar son la falta de una estrategia coherente para imponer sus intereses, una ampliación de las alianzas y el acercamiento parcial del Foro a los EE.UU.

IBSA es interpretado tanto por parte de los tres miembros como desde el exterior como un nuevo tipo de cooperación Sur-Sur, un catalizador para la formación de coaliciones multilaterales, basado en la diplomacia presidencial y con el objetivo de una alianza estratégica. El peso internacional desigual de sus miembros y (a veces) intereses divergentes con respecto a ciertos temas han llevado a que el Foro fuera caracterizado como una "alianza ad hoc" o un "menu a la carte". Por otro lado, según Gratius y John de Sousa, el Foro "está respaldado por los más altos mandos, agrega el poder, aumenta la cooperación intersectorial e inicia la creación una comunidad interregional." (Gratius y de Souza 2007: 9). IBSA es para Brasil más importante que para India y África del Sur y cumple para el líder del Cono Sur, en primer lugar, la función de coordinar intereses y aprovechar el aumento de bargaining power en organizaciones internacionales (Gomes Saraiva 2007: 57). El Foro le sirvió a Lula da Silva, además, como instrumento para su política exterior con su concentración en actuaciones multilaterales y el compromiso en favor de la democracia y los temas sociopolíticos. El anclaje de los tres países miembros de IBSA en la comunidad occidental de valores ha contribuido sustancialmente a su reconocimiento como potencias emergentes. Eso explica también el hecho de que, como Lechini subraya, "Brasil ha sido una fuerza motriz para IBSA y su política exterior es un buen ejemplo de las nuevas modalidades de la cooperación Sur-Sur más productiva." (Lechini 2007: 29). El Foro ha financiado proyectos de desarrollo en algunos de los países más pobres del mundo (Burundi, Cambodia, Guinea-Bissau, Laos, Palestina, Sierra Leona y Haití). 
El creciente protagonismo de Brasil en la OMC - resultados ambiguos

Una fuerte representación en la OMC es importante para Brasil por el hecho de que el sector exportador se ha desarrollado de manera muy positiva desde la mitad de los años 90, lo que explica su alto rango en la política exterior brasileña (Almeida 2007: 7). Como un indicador de la adhesión al principio del multilateralismo puede ser valorado, además, el hecho de que Brasil es, junto con India, uno de los países que con mayor frecuencia se ha dirigido al tribunal de arbitraje de la OMC en caso de conflictos comerciales ${ }^{2}$. Los resultados de la política brasileña en la OMC son, por otro lado, más bien ambiguos. El Itamaraty presentó en 2005 un candidato propio, Luiz Felipe de Seixas Corrêa, como Secretario General de la organización. Su competidor fue el uruguayo Carlos Pérez del Castillo. Después del fracaso de Corrêa en la primera votación, Brasil se abstuvo en la segunda ronda - un acto poco solidario hacia su país vecino. Finalmente, ganó Pascal Lamy, el candidato europeo.

Más importante que la cuestión del liderazgo en esta institución fue para Brasil, sin embargo, la formación de alianzas en la Ronda de Doha. Aquí el gobierno tuvo más éxito. Uno de los objetivos centrales de Brasil en la OMC es, según Celso Amorim, el ex canciller del gobierno de Lula, la lucha por un sistema de poder menos asimétrico y un régimen de comercio menos autocrático. Eso apunta a la reducción de subvenciones agrarias en los países desarrollados y, paralelamente, al mantenimiento de barreras proteccionistas para manufacturas en los países en desarrollo. La tensión estratégica en la política comercial brasileña se mueve entre los intereses económicos propios y la intención de ser abogado del Sur contra la agenda del Norte (de EE.UU., Japón y Europa).

En lo que sigue, quiero exponer qué rol ha jugado Brasil en la OMC durante el gobierno de Lula da Silva, diferenciando entre tres funciones: agenda setting, veto player o simple observador.

Revisando las negociaciones de la Ronda Doha, se puede decir que Brasil ha jugado un rol decisivo en ellas. Un rol líder ha tenido este país, sobre todo, en la Conferencia de Cancún en 2003, que se caracterizó por el estancamiento de las negociaciones causado por rigideces tanto del Norte como del Sur. Por una iniciativa brasileña junto con India, China y África del Sur, fue creado el Grupo de los 20. Este grupo estuvo formado inicialmente por 20 países en desarrollo y países recientemente industrializados, y en la conferencia de Cancún adoptó una estrategia confrontativa con los países del Norte, especialmente la U.E. y EE.UU.

Según Stefan A. Schirm, la acción de Brasil en Cancún es un ejemplo de liderazgo en asuntos internacionales, aceptado por los seguidores, porque se basó tanto en "benevolencia en la forma" (mostró continuidad y consistencia en sus metas políticas), como en "benevolencia en substancia", incluyen-
¿Sur-Sur contra NorteSur? La política brasileña extraregional bajo la presidencia de Lula da Silva (2002-2011)

Klaus Bodemer

\footnotetext{
2 El país ocupa al respecto el cuarto lugar, India el quinto (Hurrel 2006: 11).
} 
América Latina: cambios a nivel regional y en su inserción internacional do los intereses de los seguidores. Eso cambió en las dos sesiones siguientes de la Ronda Doha, la de Ginebra (2004) y Hong Kong (2005). En Ginebra, la posición del Grupo de los 20 fue más moderada y las negociaciones culminaron en un acuerdo marco sobre las negociaciones venideras. Brasil e India experimentaron un ascenso por la incorporación provisoria en el G5. La reunión ministerial de la OMC en Hong Kong en diciembre de 2005, sin embargo, ha mostrado intereses divergentes tanto entre el Grupo de los 20 como entre este grupo y las potencias emergentes. Mientras Brasil e India mostraron una actitud defensiva con respecto a la liberalización de sus mercados para productos elaborados del Norte, China tenía una posición contraria. Los países emergentes del Grupo de los 20 fueron sólo en parte benevolentes en la forma, pero su benevolencia en substancia se vio perjudicada por el ascenso de Brasil e India en el G5. Ambos países tenían, además, diferentes intereses económicos, persiguieron, en primer lugar, sus propios intereses nacionales y no tomaron suficientemente en cuenta los intereses de los seguidores. Una de las consecuencias de esta falta de coincidencia fue que otros países (dentro y fuera del Mercosur) no compartieron la aspiración de Brasil a ocupar el puesto del Secretario General en la OMC (Schirm 2006: 8). Lo mismo pasó con la voluntad de la diplomacia brasileña de obtener un sitio en el Consejo de Seguridad. Finalmente, el rechazo de Brasil al proyecto ALCA, confirmado en la Cumbre de las Américas en Mar del Plata en noviembre de 2005 tampoco fue aceptado por todos los posibles seguidores del liderazgo de Brasil. Eso es otro ejemplo que verifica la hipótesis de que la dificultad de Brasil para ofrecer las condiciones requeridas para la aceptación de su liderazgo por parte de los seguidores puede contribuir a explicar su fracaso parcial en uno de los objetivos centrales de su política exterior: alcanzar el liderazgo regional y un protagonismo internacional no cuestionado (Schirm 2006: 12).

\section{La política brasileña en el G20 - la necesidad de coaliciones variables}

El colapso de Lehman Brothers en 2008 y sus consecuencias desastrosas para la economía internacional han dejado al descubierto un profundo problema en la gobernanza global: la ausencia de mecanismos capaces de controlar y regular los mercados financieros globales. En vez de recurrir a soluciones unilaterales (como en la crisis de 1930) o de refugiarse en las organizaciones multilaterales de Bretton Woods (como el FMI), los gobiernos se esforzaron por buscar una solución de la crisis a través de una respuesta coordinada y negociada entre los principales líderes globales que el llamado Grupo de los 20 (G20), como foro de construcción de este consenso, hizo propia. A este grupo pertenecen no sólo los países líderes de la OCDE, sino también un grupo de países en desarrollo, considerados como economías emergentes, entre ellos Brasil. Con este nuevo Foro se esperaba contribuir a una mayor eficacia deci- 
sional y a una mayor legitimidad que la que podían brindar las instituciones establecidas en el ámbito de las Naciones Unidas (Botto, 2012: 130).

El G20 fue creado por el G8 en el año 1999, pero adquirió visibilidad y proyección global sólo en el año 2008 como consecuencia de la crisis financiera que se desató en EE.UU., pasando entonces a ser considerado el principal foro de discusión multilateral en temas económicos. El Foro ha organizado una serie de cumbres. Los mayores avances del Grupo radican, primero, en la ampliación del número de países participantes, incluyendo doce países en desarrollo que, junto con los G8 y la UE, representaban más del 74\% del PBI mundial; segundo, en la diversidad de la definición de la agenda económica global; tercero, en la promoción del diálogo entre Norte y Sur, superando así la brecha entre visiones opuestas en torno al desarrollo económico (Botto, 2012: 137); y cuarto, en la inclusión de algunas instituciones multilaterales, como el FMI, el Banco Mundial, la OMC, el Comité de Basilea y el IOSCO, organismo internacional que agrupa a los reguladores de los mercados de valores. Si hacemos un balance de lo alcanzado hasta hoy en este Foro, podemos decir que el objetivo central del G20, es decir, aumentar la eficiencia en la toma de decisiones y la legitimidad, fue alcanzado sólo parcialmente. Con sólo 19 países miembros, el G20 sigue siendo un foro de carácter excluyente. Si bien participan todos los países miembros en sus encuentros ministeriales, no necesariamente todos tienen la misma capacidad de influencia en las decisiones. Eso depende del poder económico de cada miembro, de su capacidad de formar coaliciones estables, etc. Como la experiencia muestra, aquellos países que son capaces de actuar de manera pragmática y concertada y comparten las mismas convicciones, como los de la OECD o el FMI, tienen más influencia. Con respecto a la eficiencia en la toma de decisiones, el G20 ha introducido una innovación: la existencia de una agenda de discusión abierta, en la que cada uno de los países anfitriones define el bien global sobre el cual se basará el debate en la nueva cumbre. La agenda que se construye de esta manera en las reuniones ministeriales anuales es muy variada y rica. Pero esta pluralidad tiene sus costos, porque corre el riesgo de expandir cada vez más aquellos temas que se definen como bienes globales, dificultar consensos y perder de vista el objetivo original del G20: controlar los mercados financieros. No puede sorprender, por lo tanto, que los logros en materia de coordinación y control de los flujos financieros internacionales sean hasta el momento modestos y más bien de carácter cosmético (Botto 2012: 141), y eso a pesar de los esfuerzos realizados al respecto por los miembros latinoamericanos que disponen de ciertas "ventajas" en esta materia. Frente a las propias experiencias de la "década perdida" y los programas neoliberales de ajuste, los tres miembros latinoamericanos del G20 - Brasil, México y Argentina - están en mejores condiciones que otros para proponer y focalizar la discusión sobre los peligros de flujos financieros no controlados, las políticas de desregulación financiera y un modelo de desarrollo librado a las fuerzas del mercado. Un segundo elemento positivo
¿Sur-Sur contra Norte-

Sur? La política brasileña

extraregional bajo la presidencia de Lula da Silva (2002-2011)

Klaus Bodemer 
América Latina: cambios a nivel regional y en su inserción internacional del que disponen los miembros latinoamericanos es la rica experiencia de la región en la coordinación e integración desde los años 90. Sin embargo, el hecho de que el avance en la coordinación e integración, incluso en la creación de nuevos regímenes como ALBA, UNASUR y CELAC, ha provocado, al mismo tiempo, una mayor fragmentación de la región es una de las causas de que la conciliación entre lo regional y lo multilateral se encuentre todavía en un estado embrionario y que muchas veces sigan predominando posiciones nacionales en los debates del G20, lo cual constituye un reflejo de legados históricos y niveles de desarrollo diferentes y del peso de asuntos domésticos en los países miembros. Una posición latinoamericana común es, por lo tanto, muchas veces difícil de lograr y suele reducirse a ponerse de acuerdo en el mínimo denominador común. Una concertación entre los tres miembros latinoamericanos antes de las reuniones es más bien la excepción que la regla. Es más: ninguna posición concertada entre los tres miembros latinoamericanos puede reclamar representar a toda la región.

Los países emergentes tuvieron, sin embargo, cierto poder de establecer la agenda cuando fueron capaces de formar coaliciones u obtener la presidencia de los encuentros como, por ejemplo, México en la Cumbre de México en junio de 2012 (Gnath y Schmucker 2011). En las primeras cumbres, en las cuales se buscaron respuestas inmediatas y coordinadas y paquetes de rescate frente a la crisis financiera internacional, los miembros del Norte y del Sur encontraron respuestas ampliamente concertadas, incrementando los fondos internacionales y programas nacionales de estímulo al crecimiento. Surgieron, sin embargo, discrepancias con respecto a dos materias:

(1) Con respecto al reparto de la carga de los costos surgieron discrepancias entre los participantes del Sur y se formaron coaliciones que cruzaron las fronteras Norte-Sur. Brasil estaba en contra de la posición europea de que los bancos debían co-participar en el pago, por ejemplo, por medio de un impuesto a las transacciones financieras (Tobin Tax), alineándose con la posición de Canadá. Como China e India, los brasileños argumentaron que sus bancos habían sido sólidos durante la crisis y que, por lo tanto, no era serio imponerles un impuesto. De esta manera junto con Canadá, otro poder de veto, los poderes emergentes China, India y Brasil lograron vetar este tema en la cumbre (Gnath y Schmucker 2011: 8 y s.).

(2) Un segundo tema central en las cumbres de G20 fue la reforma de la regulación financiera. EE.UU. y la U.E. fueron fuertes defensores de una mayor regulación financiera como se había previsto en Basilea III. Brasil y los otros países emergentes apoyaron esta posición, pero no han dado a este asunto la misma prioridad que al desarrollo, un objetivo que fue lanzado en la Cumbre de Pittsburgh (2009) con el "Programa marco de referencia para un crecimiento fuerte, sostenible y equilibrado" (Framework for Strong, Sustainable and Balanced Growth) (G20 2010). China apoyó este programa, pero define el término unbalanced growth más como "desequilibrio global" que como déficits (o superávits) comerciales. Brasil, por su parte, estaba en contra de 
medidas de estimulación económica a costa del crecimiento, argumentando que las economías de los países emergentes no deberían ser cargadas por medidas de recuperación global y que los países exportadores del Norte no deberían implementar programas severos de ajuste financiero a costa de los países emergentes. China, finalmente, consideraba su política monetaria como un asunto doméstico y rechazó el involucramiento del G20 en este terreno (Gnath y Schmucker 2011: 9-13).

El consenso más amplio entre el Norte y el Sur en las Cumbres del G20 se refirió a un tercer tema, la reforma de las instituciones financieras internacionales. Como resultado, en octubre de 2010, los Ministros de Hacienda del G20 acordaron un paquete de reformas en la Cumbre de Seúl bajo la gestión de la delegación coreana y del Directorio Ejecutivo del FMI. El cociente adjudicado a los votos en el directorio fue modificado en un $6 \%$ en favor de los grandes países emergentes. China ocupó el tercer rango después de EE.UU. y Japón, reemplazando a Alemania que disponía de menos del $6 \%$ de los votos y se posicionó en el cuarto lugar. Los diez accionistas más poderosos en el FMI incluyeron a Rusia, India y Brasil. La cumbre del G20 acordó, además, una reforma del Directorio Ejecutivo del FMI. Los países europeos avanzados debieron renunciar a 2 de sus 8 cargos. El Directorio fue ampliado de 20 a 24 cargos en contra de la posición de EE.UU. de reducirlo. (Gnath y Schmucker 2011: 14).

En la Cumbre de Seúl, en 2010, se acordó, además, el “Consenso de desarrollo para el crecimiento compartido" (Development Consensus for Shared Growth), declarando que no hay una fórmula única para el éxito del desarrollo. Esta vuelta hacia el desarrollo fue apoyada fuertemente por los países emergentes y en desarrollo. A pesar del hecho de que esa no era la prioridad de China, este país abogó en esta cumbre por una atención mayor hacia el mundo en desarrollo y por la promoción del desarrollo global en el contexto del G20 (YanG2010: 23).

Finalmente, Brasil y los otros países emergentes han salido de la Cumbre del G20 en Cannes en noviembre de 2011 muy fortalecidos frente a las divisiones europeas y la debilidad de EE.UU. Dilma Rousseff atacó las medidas de ajuste, pues dijo que "Brasil tiene una experiencia exitosa a la hora de enfrentar una crisis con inclusión social y generación de empleos. La inclusión de 40 millones de personas en la clase media fue no solamente un imperativo moral sino una cuestión de eficiencia política" (Núñez 2011: 1).

Los europeos querían que los emergentes invirtieran en un Fondo Europeo de Estabilidad Financiera, pero Brasil y el resto de los BRICS encabezados por China prefirieron aportar al FMI y no a tal Fondo. Esta apuesta por el FMI fue para los emergentes y para Brasil una jugada con connotaciones geopolíticas, ya que les hará volver a ganar poder en la próxima revisión de cuotas del Fondo, algo a que se resiste EE.UU. Rousseff explicó su posición comentando que "el Fondo tiene la responsabilidad de actuar frente al riesgo de todo el sistema y no sólo ante la crisis europea, por lo que entendemos que es importante que tenga más recursos. Y Brasil está dispuesto a participar porque esos recursos tienen la garantía del FMI y, al mismo tiempo, tienen una
¿Sur-Sur contra Norte-

Sur? La política brasileña

extraregional bajo la

presidencia de Lula

da Silva (2002-2011)

Klaus Bodemer 
América Latina: cambios a nivel regional y en su inserción internacional característica que para nosotros es muy importante: tiene reglas”. Brasil, según Rousseff, está dispuesto a realizar una contribución al FMI mediante "acciones bilaterales" para aumentar su capacidad de préstamo anual. "Una ampliación del FMI en momentos de crisis es muy importante para reducir riesgos sistémicos" (Núñez 2011: 1).

Haciendo un balance del comportamiento de los países emergentes y, entre ellos, de Brasil en el G20 y respondiendo a nuestra pregunta original de si los tres países emergentes China, India y Brasil han actuado como agenda setters, veto players o simples observadores en el G20, podemos decir que - si bien comparten algunas preferencias generales, en primer lugar, con respecto a lograr una voz más fuerte en la gobernanza económica global - resulta difícil para ellos alcanzar una posición común en este Foro debido a sus diferentes tradiciones en políticas económicas, a los diferentes desafíos económicos que debe enfrentar cada uno y a sus preferencias variables en una amplia gama de temas del G20, por ejemplo, en la política cambiaria o el área de la supervisión financiera. Estas divergencias son, en realidad, entre los países emergentes mucho más grandes que entre los países europeos o los miembros del G8 dentro del G20 (Gnath y Schmucker 2011: 17 y s.). En suma, un análisis de los resultados de las Cumbres del G20 y de las preferencias de sus integrantes sugiere que los países emergentes no han sido capaces de influir (o modificar) la agenda formal de los líderes del G20 con posiciones comunes. Sin embargo, mientras los EE.UU. y los miembros europeos del G20 han dominado la agenda de las primeras cumbres del G20, los miembros del G8 no han actuado necesariamente de manera concertada. La "cacofonía" de las voces del G8 ha dado a los países miembros emergentes un espacio de maniobra en el G20. Como consecuencia, los países emergentes de peso no fueron simples observadores en las Cumbres del G20. Su capacidad de influir en los resultados dependió de su habilidad de formar coaliciones entre ellos y con los países del G8. Una lectura adicional de las cumbres es que la línea divisoria tradicional entre el Norte y el Sur ya no corresponde a la realidad, es decir, que surgen coaliciones variables según los temas. Lo esencial para llegar a consensos es que los países emergentes permanezcan comprometidos con el proceso del G20 y que no surja una división rígida entre los G8 y los países emergentes. Para evitar que se abra una brecha, va a ser importante incluir en la agenda de las Cumbres futuras aquellos temas que sean de interés tanto para los países industrializados como para los emergentes (Gnath y Schmucker 2011: 18). Finalmente, teniendo en cuenta a la mayoría de los países en desarrollo que están representados en la Asamblea General de la ONU, la postura de que los países emergentes representan al Sur en el G20 es fuertemente cuestionada, porque ni Brasil ni China ni India ni África del Sur están concertando su política exterior y sus posiciones en este grupo con otros países del Sur. Más aún, como indica Eduardo Gudynas, la revalorización del G20 puede aumentar las asimetrías en el sistema internacional y erosionar el rol de la ONU y la búsqueda de un desarrollo alternativo (Gudynas 2012). 


\section{El rol de Brasil en los BRICS}

El acrónimo BRIC (más tarde BRICS, a partir del ingreso de Sudáfrica el 24 de diciembre de 2005) fue acuñado en el año 2001 en el espacio de las agencias calificadoras de riesgo y popularizado por el economista Jim O'Neill de Goldman Sachs (O’Neill 2001). El término junta los grandes países emergentes del siglo XXI bajo una etiqueta. Se trata de cinco naciones de cuatro continentes (Brasil, Rusia, India, China y África del Sur). Estos países no disponen de una coherencia histórica, tienen sistemas políticos competitivos e intereses geoestratégicos diferentes. Los BRICS reúnen en conjunto un potencial enorme: el $43 \%$ de la población mundial, el 30\% del crecimiento mundial entre 2000 y 2006, la duplicación de su participación en el comercio mundial en el mismo período y, finalmente, el $35 \%$ de las divisas mundiales.

Desde su formación como agrupación política, los BRICS se han constituido en un importante actor internacional. El primer intento de articulación política de los BRICS se remonta a 2009, cuando se celebró su primera Cumbre en Ekaterimburgo, Rusia. Tanto aquí como en las cumbres anuales siguientes, el grupo abogó en favor del fortalecimiento del orden multipolar y, en particular, de la reforma del sistema financiero mundial bajo cuatro principios rectores: (1) toma de decisiones y procesos de implementación democráticos $y$ transparentes en las organizaciones financieras internacionales, (2) una base jurídica sólida, (3) la compatibilidad entre las actividades de las instituciones de regulación nacionales y los organismos internacionales, (4) el fortalecimiento de los mecanismos de gestión de riesgos y de supervisión (Esteves, 2012: 152). Desde el encuentro en Rusia, los Jefes de Estado de los BRICS se reunieron anualmente y promulgaron declaraciones conjuntas que generaron iniciativas en diversas áreas y dieron lugar a una serie de acuerdos de cooperación en los más diversos sectores de la acción gubernamental (véanse los detalles en Esteves 2012: 153 y ss.).

En la última Cumbre en Delhi, en marzo 2012, se decidió instalar instituciones paralelas a las tradicionales, dominadas por los países del Norte y, como primer paso, se creó un banco de desarrollo que sólo actúa con créditos en las monedas de los BRICS. Según sus fundadores, este banco debería difundir conceptos de desarrollo propios, por ejemplo, de China o India a Indonesia o Nigeria, como alternativa a las recetas del Banco Mundial.

Los BRICS no son sólo un grupo de "países emergentes", sino que, dada la presencia de Rusia, configuran más bien una agrupación sui generis que cuestiona el status quo y promueve una revisión de las prerrogativas vigentes en el orden internacional. Sin embargo, los miembros del grupo comparten pocas posiciones de consenso dentro de sus agendas, lo cual es consecuencia de la gran asimetría de poder existente dentro de él y de la heterogeneidad de sus preferencias. Esta heterogeneidad se muestra tanto en el área de seguridad, donde China y Rusia defienden el status quo mientras que Brasil, India y Sudáfrica lo cuestionan, como en la de comercio y la de medio ambiente, tres agendas mul-
¿Sur-Sur contra Norte-

Sur? La política brasileña

extraregional bajo la

presidencia de Lula

da Silva (2002-2011)

Klaus Bodemer 
América Latina: cambios a nivel regional y en su inserción internacional tilaterales del primer rango (Esteves 2012: 166-176). Los BRICS encuentran, por lo tanto, grandes dificultades para la formulación de propuestas substantivas para la reforma de las principales instituciones y regímenes multilaterales. La importancia de esta agrupación va, sin embargo, más allá de una proyección internacional, pues incluye elementos de cooperación Sur-Sur de nueva índole. Su filosofía básica es aprovechar las propias experiencias e intercambiarlas en vez de imitar recetas importadas desde el Norte ("Die Zeit" 2012). Según sus miembros, BRICS se comprende como el primer foro mundial sin participación de las potencias occidentales, su hora de nacimiento es la crisis financiera en estos países, su misión es tomar distancia de los grandes conflictos, es decir, no hacer geopolítica sino sacar millones de la miseria. Es éste el campo donde se puede esperar resultados más significativos. Tanto Brasil, como China han implementado amplios programas de cooperación Sur-Sur en los años recientes, distanciándose de la tradicional cooperación al desarrollo Norte-Sur, su paternalismo y sus rígidos criterios de eficiencia. Si estos proyectos realmente cumplen los criterios de no injerencia, simetría y horizontalidad que sus protagonistas prometen, es algo que queda por verse en el futuro. Ante el hecho de que hasta ahora los países del Norte mostraron poca disponibilidad a entrar en diálogo con los BRICS y de que el grupo no cuenta con una institucionalización, queda abierta la cuestión de si los países BRICS como conjunto van a ser realmente la potencia económica líder hacia 2050, como pronostican algunos expertos.

Esfuerzos brasileños frustrados en el Consejo de Seguridad de la ONU, pero activa participación en misiones de paz

Desde 1994, Brasil hace una campaña por ser reconocido como miembro permanente en el Consejo de Seguridad. A este proyecto se le otorgó una alta prioridad bajo Lula da Silva. El Itamaraty persigue este plan en el marco del G4, compuesto por Brasil, Alemania, Japón e India (Lima y Hirst 2006: 29). El presidente brasileño argumentó que la ampliación significaría una democratización del Consejo de Seguridad (Ministério das Relações Exteriores 2008: 206), lo que en realidad no es muy convincente, porque la ampliación significaría nada más que un fortalecimiento de algunos países líderes regionales. Tampoco es convincente el argumento de que Brasil hace un aporte muy importante a la ONU, porque este país paga sólo el 1,5\% (México 1,9\%; Japón 19\% y Alemania 8,7\%) (Schirm 2007: 10 y s.). Mientras China es renuente al objetivo de Brasil (y de Japón) de ser miembro permanente, Rusia se ha pronunciado favorablemente por la candidatura brasileña. Las ambiciones de Sudáfrica al respecto chocan con las pretensiones de Nigeria y Egipto a que los africanos ocupen un asiento permanente de manera rotativa. La misma propuesta fue hecha por Argentina y México para América Latina a mediados de la década para frenar las ambiciones de Brasil. Más peso tiene, por otro lado, el compromiso de Brasil en las misiones de paz, como, por ejemplo, su 
conducción de la misión MINUSTAH en Haití. Ya en años anteriores Brasil había participado con fuerzas policiales en una misión de mantenimiento de la paz en Timor del Este y en misiones de observación de la ONU en El Salvador y Mozambique y ha enviado 1.300 soldados a una misión de la ONU en Angola. Brasil es, además, un destacado participante en los mecanismos regionales de seguridad colectiva en el marco de la SADC (Comunidad para el Desarrollo de África), desde su ingreso a la organización después del fin del apartheid. Con todo eso, Brasil demuestra su compromiso como potencia de ordenamiento y su adhesión a la comunidad occidental de valores.

\section{¿Es la creciente proyección extraregional de Brasil un éxito? - algunas conclusiones}

Desde la presidencia de Cardoso y, con más fuerza, desde el primer gobierno de Lula da Silva, la política exterior brasileña ha reclamado una importante posición y un peso mayor en la arena regional e internacional, mostrando, en general, un comportamiento cooperativo y orientándose al principio del multilateralismo, es decir, del reconocimiento de instituciones y foros internacionales como mecanismos centrales de articulación y agregación de intereses y de solución de conflictos, demostrando así su adhesión a la comunidad occidental de valores (Lima y Hirst 2006; Vigevani y Ramazini Jr. 2009). En este contexto, es una expresión de cierto pragmatismo que la diplomacia brasileña comprende y practica su cooperación activa con países del Sur no - según la lógica del juego de suma cero - como ataque al Norte, sino como un complemento, lo que le permite formar a veces coaliciones ad hoc con países industrializados, si existen intereses convergentes, por ejemplo, en el marco del G4 con Japón y Alemania y en el G20 en algunos temas seleccionados.

Brasil forma hoy parte de aquellos países que hacen un reclamo específico de participación en el sistema internacional, disponen de la capacidad de articular este reclamo y son por lo tanto categorizados con razón como potencias emergentes (Schirm 2006). No existen, sin embargo, criterios unívocos que permitan una adscripción clara a este grupo. Stefan A. Schirm ha elaborado algunos años atrás un catálogo de criterios que me parecen útiles para clasificar los países emergentes según su poder posicional y su capacidad de influencia:

1. El país debe reclamar para sí un rol relevante en el ámbito internacional y disponer de los recursos políticos y económicos necesarios para llevarlo a la práctica.

2. El país debe llevar a cabo actividades que subrayen y concreticen este reclamo.

3. El país es reconocido y aceptado por otros actores internacionales como importante actor y líder.

4. El país tiene influencia real en el acontecer internacional, en el sentido de power of outcomes, (Schirm 2007: 2 y s.).
¿Sur-Sur contra Norte-

Sur? La política brasileña

extraregional bajo la presidencia de Lula da Silva (2002-2011)

Klaus Bodemer 
América Latina: cambios a nivel regional y en su inserción internacional
Existe un consenso en la bibliografía especializada acerca de que, desde el gobierno de Lula, Brasil intenta jugar un rol internacional diferente que en años anteriores y que ha desarrollado, por lo tanto, una amplia gama de actividades (Zilla 2009; Almeida 2007; Flemes 2007) que le permiten un mayor protagonismo, tanto regional como internacional. Hay también acuerdo en que el país dispone de los recursos económicos y políticos necesarios para tener cierto éxito al respecto (criterio 1 y 2). Brasil es también cada vez más reconocido como un actor de peso, tanto político como económico, en el ámbito internacional. Eso, sin embargo, no implica automáticamente el reconocimiento de un rol de liderazgo (criterio 3). Poder posicional no se traduce automáticamente en poder de influencia. El power of outcome del país es, como intentamos demostrar en este trabajo, más bien modesto (criterio 4). Al prestigio y la influencia internacional han aportado también sus éxitos internos, es decir, la incorporación de alrededor de 40 millones de pobres en la clase mediabaja. Un instrumento útil de la cooperación Sur-Sur fue la formación de y la participación activa en alianzas, por ejemplo en IBSA, donde ha jugado un rol destacado, sobre todo en la cooperación económica. Esta cooperación le ha permitido a Brasil encontrar nuevos socios comerciales y explorar nuevos mercados para sus productos (Gomes Saraiva 2007: 56). En favor de los socios más débiles, sobre todo en África, Brasil intenta, además, presentarse como "abogado del Sur" - lo que es, como vimos, por lo menos, discutible - y espera como contrapartida el apoyo para sus propios proyectos políticos (Seitenfus 2007; Lechini 2007). La diplomacia de Lula ha apuntado, además, exitosamente a lograr una mayor independencia del país y un mayor margen de maniobra y de decisión por medio de la diversificación de los socios externos (Vigevani y Cepaluni 2007; Marinho Nogeira 2008). El método de acción a nivel extraregional no se diferencia del que emplea a nivel subregional y regional, es decir, que el país privilegia una actitud coordinada y consensuada con los países emergentes más fuertes y reclama un liderazgo ante los países más débiles, sin entrar, por otro lado, en una competencia fuerte con otros países líderes como, por ejemplo, con India y China por sus actividades respectivas en África - para no arriesgar la necesaria cooperación con ellos. Tanto la cooperación como el reclamo de liderazgo fueron, en gran parte, area-oriented en el sentido de alianzas ad hoc. Sin embargo, durante los años de Lula, ni la cooperación con system-affecting states ni con non-system-affecting states fuera de la región fue tan intensa como a nivel regional (Seifert 2009: 81).

En suma, el balance con respecto a los criterios de aceptación (criterio 3), influencia y power of outcome (criterio 4) es más ambivalente que con respecto a los criterios 1 y 2. La mayoría de los países de la región reconocen la relevancia y el peso destacado de Brasil en la región y la arena internacional, y también su rol como mediador en conflictos, pero siguen siendo más bien escépticos con respecto a su reclamo de ser el vocero y/o representante del Sur, a pesar de su estrategia de cortejo. El reconocimiento de Brasil como socio importante se produce, por ejemplo en el G20 y la OMC, sólo en el caso de intereses conver- 
gentes (Lechini 2007). El balance con respecto a su power of outcome (criterio 4) es en parte negativo, en parte positivo. Ni sus esfuerzos por obtener una representación permanente en el Consejo de Seguridad ni por alcanzar mayor poder de decisión en el FMI fueron exitosos hasta hoy. De la misma manera fracasó su candidato para el puesto del Director General de la OMC. En este caso, su "egoísmo", es decir, su abstención en la segunda votación (a costo del candidato uruguayo) ha lesionado además su imagen en la región y en otros países del Sur (Burges 2007: 1352). Un éxito fue, por otro lado, su candidatura para la conducción de la FAO, a costo del candidato de su rival México. Un cierto éxito fue, además, la formación de alianzas en la OMC y (algo menos) en el G20, lo que significa una ganancia de poder de negociación y el reconocimiento de Brasil como un importante tomador de decisiones (Burges 2007; Lima y Hirst 2006). También es un éxito el hecho de que la diplomacia brasileña haya avanzado en extender la cooperación Sur-Sur sin por eso empeorar las relaciones del país con los países industrializados, sino más bien contrabalancearlas (Seitenfus 2007: 16). Un indicador elocuente al respecto es el hecho de que la U.E. y Brasil han establecido en 2007 una "alianza estratégica" y que Brasil ha cambiado desde la Conferencia de Copenhague su posición rígida y confrontativa respecto al tema del medio ambiente y el cambio climático.

A pesar de estos avances, existen también trampas en la profundización de la cooperación Sur-Sur, porque existe el peligro de que se reproduzca también en el Sur la estructura desigual del comercio que existe entre Norte y Sur. China, por ejemplo, podría asumir el rol de país exportador de manufacturas, mientras Brasil permanecería como proveedor de materias primas. Inversa sería su relación con los países de la región subsahariana (Schmalz 2005: 2).

Según las proyecciones de Goldman Sachs (2010), la producción económica total de los BRICS superará a la de los países del G7 en 2035. Con sus socios BRICS, Brasil puede ser, alrededor de 2040, una potencia grande, superando a las grandes potencias de Norte en términos de capacidades materiales. Pero a diferencia de sus competidores China, India y Rusia, las alianzas de Brasil son significativamente predeterminadas. El país continuará siendo una potencia occidental, estrechamente vinculada con EE.UU. y Europa Occidental. Su proyección de poder es, independientemente del aumento de su presupuesto militar y de defensa en tiempos recientes, fundamentalmente la de un soft power y depende ampliamente de la calidad de sus instituciones democráticas. La mayor oportunidad que se le ofrece a Brasil a largo plazo para posicionarse en el ámbito internacional es la de una potencia ambiental emergente. Como poseedor de la selva tropical más grande del mundo, una de las mayores reservas de agua potable, la mayor bio-diversidad del planeta, la mejor matriz de energía entre los grandes países y la más exitosa producción de bio-energía, Brasil tiene los recursos para jugar este rol, si adopta las políticas necesarias para preservar estos activos y si sabe utilizarlos como instrumentos políticos en un mundo crecientemente preocupado por el cambio climático (Sotero y Elliot Armijo 2007: 43 y s.; Nolte y Stolte 2007).
¿Sur-Sur contra NorteSur? La política brasileña extraregional bajo la presidencia de Lula da Silva (2002-2011)

Klaus Bodemer 
América Latina: cambios a nivel regional y en su inserción internacional
A pesar de los destacables recursos de poder de que dispone el país, quedan algunos elementos que obscurecen su imagen de potencia de primera liga. Brasil es el único país de BRICS que, a pesar del desarrollo avanzado de la tecnología nuclear, no posee armas nucleares y cuyas tropas no superan un millón de soldados. Su estructura productiva se concentra (con las excepciones mencionadas), en gran parte, en el sector primario y agroexportador, y su contribución al comercio mundial es inferior a la de los grandes países industrializados y las principales potencias emergentes. Sus tasas de crecimiento son modestas, comparadas con las de Asia. Su sistema de educación está muy lejos de los de los otros BRICS. Ningún científico brasileño ha ganado un premio Nobel y ninguna universidad del país se encuentra entre las más sofisticadas del mundo. El columnista Andrés Oppenheimer del diario El Nuevo Heraldo, Miami, ha agregado, finalmente, otra carencia que Brasil comparte con otras sociedades latinoamericanas: la falta de una cierta "paranoia constructiva" muy difundida en los países asiáticos y en algunos países de Europa del Este (como Polonia y Chequia), es decir, una política ofensiva en ciencia, educación y desarrollo tecnológico que apunta a una mayor competitividad (Oppenheimer 2010). Hay, por otro lado, señales prometedoras al respecto en tiempos recientes. Con los objetivos del movimiento "Todos por la educación" (2007), que luego fueron adoptados por el gobierno, la creación del Observatorio Educativo, sus universidades públicas de excelencia y sus subsidios escolares (Plan Bolsa Familia y Bolsa Escuela), el país se encuentra a la punta de América Latina. Siguen existiendo, sin embargo, carencias importantes en el sector educativo, como la modesta internacionalización de las universidades, la baja sintonía entre el sector productivo y el académico, y el bajo nivel de la educación primaria y secundaria. El país es, como Andrés Oppenheimer resume bien, un "gigante tecnológico con pies en barro", "está mal, pero va bien" (Oppenheimer 2010: 268 y s.; Oppenheimer 2011), es decir, que se encuentra en un buen camino, pero todavía le queda bastante por andar para llegar al nivel de sus competidores China e India.

\section{Bibliografía}

Alden Ch., Vieira M. A. (2007), Marco, La nueva diplomacia del sur: Brasil, Sudáfrica, India y el trilateralismo, en: India, Brasil y Sudáfrica. El impacto de las nuevas potencias regionales, J. Tokatlian (comp.), Libros del Zorzal, Buenos Aires, pp.137-168.

Almeida de P. R. (2006), Uma nova arquitetura diplomática? - Interpretações divergentes sobre a política externa do governo Lula (2003-2006), "Revista Brasileira de Política Internacional", vol. 49, no 1, pp. 95-116.

Almeida de P. R. (2007), Brazil as a regional player and an emerging global power, FES Briefing Paper 8, July.

Baumann R. (2010), Brazilian external sector so far in the 21st century, "Revista Brasileira de Política Internacional", vol. 3, pp. 33-53. 
Bernal-Meza R. (2008), La política exterior del Brasil: Claves para entender las diferencias con Argentina, "Densidades", no 2, octubre, pp. 25-41.

Bernal-Meza R. (2010), International Thought in the Lula Era, "Revista Brasileira de Política Internacional", vol. 53, Special edition, pp. 193-213.

Botto M. (2012), Nuevas formas de multilateralismo. El G20: Una oportunidad para América Latina?, en: América Latina y el Caribe: Vínculos globales en un contexto multilateral complejo, F. Rojas Aravena (ed.), FLACSO, CIDOB, Teseo, Buenos Aires, pp. 129-149.

Burges S. (2007), Building a Southern Global Coalition: the competing approaches of Brazil's Lula and Venezuela's Chávez, "The Third World Quarterly", 28 (7), pp. 1343-1358.

Burges S. (2008), Consensual Hegemony: Theorizing Brazilian Foreign Policy after the Cold War, "International Relations", 22 (1), pp. 65-84.

Cervo Amado L. (2008), Inserção internacional. Formação dos conceitos brasileiros, Ed. Saraiva, São Paulo.

Cervo Amado L. (2010), Brazil's Rise on the international Scene. Brazil and the World, "Revista Brasileira de Política Internacional", vol. 53, pp. 7-32.

Declaración de Brasilia (2010), el 6 de mayo de 2003, Foro India, Brasil, Sudáfrica (IBSA), "Relaciones Internacionales", GHERI-UAM, no 45, Octubre, pp. 151-156.

"Die Zeit" (2012), Brasilien, Indien, China: Was wollen die neuen Großmächte?, "Die Zeit", $14,29.03$, p. 12.

Duarte Villa R. A., Trindade Viana M. (2010), Security issues during Lula's administration: from the reactive to the assertive approach, "Revista Brasileira de Política Internacional", vol. 53, pp. 91-114.

Esteves P. (2012), Brasil, los BRICS y la Agenda multilateral en el siglo XXI, en: América Latina y el Caribe: Vínculos globales en un contexto multilateral complejo, F. Rojas Aravena (ed.), FLACSO-CIDOB, Editorial Teseo, Buenos Aires, pp. 151-178.

Facundes Visentini P. G., Reis da Silva A. L. (2010), Brazil and the Economic, Political and Environmental Multilateralism: the Lula years (2003-2010), "Revista Brasileira de Política International", vol. 53, pp. 54-72.

Flemes D. (2007), Brasil - Regionalmacht mit globalen Ambitionen, GIGA Focus 6, pp. 1-8.

Freitas Rodrigues de D. (2010), Cooperação horizontal Sul-Sul: arranjos de concertação política entre a Índia, o Brasil a África do Sul, "Revista Brasileira de Política Internacional", vol. 53, no 1, pp. 45-66.

G20 (2010), Framework for Strong, Sustainable and Balanced Growth, The Seoul Summit Document, disponible en: www.mofa.gov.jg/olicy/economy/g20_summit/2010-2/document.pdf

Gnath K., Schmucker C. (2011), The Role of the Emerging Countries in the G20: AgendaSetter, Veto-Player or Spectator?", Bruges Regional Integration \& Global Governance Papers", 2.

Goldman S. (2010), Equity in two decades: A Changing Landscape, "Goldman Sachs Global Economics Paper", 204, New York.

Gomes Saraiva M. (2007), As estratégias de cooperação Sul-Sul nos marcos da política externa brasileira de 1993 a 2007, "Revista Brasileira de Política Internacional", vol. 50, no 2, pp. 42-59.

Gratius S., John de Souza S. L. (2007), IBSA: An international actor and partner for the EU?, Fundación para las Relaciones Internacionales y el Diálogo Exterior - FRIDE, FRIDE Activity Brief, 17 de octubre.
¿Sur-Sur contra NorteSur? La política brasileña extraregional bajo la presidencia de Lula da Silva (2002-2011)

Klaus Bodemer 
América Latina: cambios a nivel regional y en su inserción internacional
Gudynas E. (2012), After a Chinese Visit. Questions about G20: One circumstance and two problems, Guest Blogger, Triple Crisis, July 9, disponible en: http://triplecrisis.com/after_a chinese_visit_questions-about_the_g20_one_-circumstance_and_two_problems/more-6432

Hurrell A. (2006), Hegemony, liberalism, and global order: what space for would-be great powers?, "International Affairs", 82 (1), pp. 1-18.

Katzenstein P. (2005), A World of Regions: Asia and Europe in the American Imperium, Ithaca: Cornell University Press.

Lechini G. (2007), Middle Powers, IBSA and the New South-South Cooperation Report: The Multipolar Movement, NACLA, North American Congress on Latin America.

Lessa A. C. (2010), Brazil's strategic partnerships: an assessment of the Lula era (2003-2010), "Revista Brasileira de Política Internacional", año 53, Special Edition, pp. 115-131.

Lima Soares M. R., Hirst M. (2006), Brazil as an intermediate state and regional poweraction choice and responsibilities, "International Affairs", 82 (1), pp. 21-40.

Malamud A. (2010), A leader without followers? The growing divergences between the regional and global performance of Brazilian foreign policy, Paper prepared for delivery at the $7^{\text {th }}$ Pan-European International Relations Conference (SGGIR-ECPR), Stockholm, September 9-11.

Marinho Nogueira J. L. (2008), A inflexão da política externa brasileira para o Sul e o Fórum IBAS. Análise Desenvolvimento/Integração Regional, Pontifícia Universidade Católica de Minas Gerais - PUC Minas, disponible en: www.puc.minas.br/conjuntura

Ministério das Relações Exteriores do Brasil (2008), Brazilian Foreign Policy Handbook, Brasilia.

Nolte D., Stolte Ch. (2007), Machtressource Bioenergie. Eine neue strategische Partnerschaft zwischen Brasilien und den USA, GIGA-Focus, no 3.

Núñez R. (2011), México preside un G20 donde los emergentes (Brasil) ganan aún más peso, "Infolatam", 8 de noviembre, disponible en: http://www.infolatam.com/2011/11/08/ mexico-preside-un-g-20-donde-los-emergentes-brasil-ganan-aun-mas-peso/

Oliveira Fernandes de M. (2005), Alianças e coalizóes internacionais do governo Lula: o Ibas e o G20, "Revista Brasileira de Política Internacional", 8 (2), pp. 55-69.

O'Neill J. (2001), Building better global economic BRICs, "Goldman Sachs Global Economics Paper", 66, October.

Oppenheimer A. (2010), ¡Basta de historias!: La obsesión latinoamericana con el pasado $y$ las 12 claves del futuro, Editorial Sudamericana, Buenos Aires.

Oppenheimer A. (2011), Nos falta la paranoia constructiva, "Portafolio", 21 de febrero, disponible en: http://www.portafolio.co/economía/nosfalta_la_paranoia_constructiva

Pedersen T. (2002), Cooperative hegemony: power, ideas and institutions in regional integration, "Review of International Studies", no 28, pp. 677-696.

Santos Dalci dos M. (2011), Actividades prospectivas en Brasil: pasado, presente y futuros posibles, "Ekonomiaz", no 76, 1.er cuatrimestre, pp. 190-211.

Schmalz S. (2005), Lula goes South. Brasilien als Vorreiter zu einer neuen Welthandelsorganisation?, "Lateinamerika Nachrichten", 373/374, Juli-August.

Schirm S. A. (2006), Leadership in Regional and Global Politics: Why do Emerging Powers (sometimes) Fail to Reach Their Goals?, la ponencia elaborada para el taller "The Rise of (New) Regional Powers in Global and Regional Politics", GIGA, Hamburg, December 11-12. 
Schirm S. A. (2007), Die Rolle Brasiliens in der globalen Strukturpolitik, DIE, "Discussion Paper", 16.

Seitenfus R (2007), O Brasil e suas relações internacionais, “Carta Internacional”, 2 (1), Marzo, pp. 11-21.

Seifert J. (2009), Die strategische Funktion der Süd-Süd-Kooperation in der Außenpolitik Brasiliens, Master of Art, Tübingen.

Sotero P. Elliot Armijo L. (2007), To be or not to be a BRIC?, "Asia Perspective", vol. 31, no 4, pp. 43-70.

Vigevani T., Cepaluni G. (2007), A Política Externa de Lula da Silva: A Estratégia da Autonomia pela Diversificação, "Contexto Internacional”, 29 (2), pp. 273-335.

Vigevani T., Ramanzini Jr. H. (2009), Brasil en el centro de la integración. Los cambios internacionales y su influencia en la percepción brasileña de la integración, "Nueva Sociedad", no 219, pp. 76-96.

Viola E., Pio C. (2003), Doutrinarismo e Realismo na Percepção do Interesse Nacional: Política Macroeconômica, Segurança e ALCA na relação Brasil - Estados Unidos, en: Brasil e EUA no novo milenio, M. G. Oliveira (org.), NEA/ Ed. Universitaria da UFPE, Recife, pp. 13-42.

White L. (2006), IBSA: A State of the Art. Conferencia Poderes emergentes y seguridad regional: el caso IBSA, Universidad San Andrés, Buenos Aires.

Yang J. (2010), Country Fact Sheet - China, en: The G20: a "Global Economic Government" in the Making? International Policy Analysis, S. Pohlmann, S. Reichert, H. René Schillinger (ed.), Friedrich Ebert-Stiftung.

Zilla C. (2009), Brasilien: Eine Regionalmacht mit globalen Ansprüchen, en: Neue Führungsmächte: Partner deutscher Außenpolitik?, J. Husar, G. Maihold; S. Mair (ed.), Nomos, Baden-Baden, pp. 49-67.
¿Sur-Sur contra Norte-

Sur? La política brasileña

extraregional bajo la

presidencia de Lula

da Silva (2002-2011)

Klaus Bodemer 